ARTICLE

https://doi.org/10.1038/s41467-019-11980-6

\title{
Potent antibody lineage against malaria transmission elicited by human vaccination with Pfs25
}

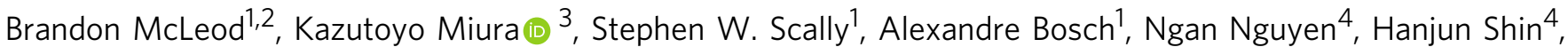
Dongkyoon Kim ${ }^{4}$, Wayne Volkmuth ${ }^{4}$, Sebastian Rämisch (10 ${ }^{5}$, Jessica A. Chichester ${ }^{6}$, Stephen Streatfield ${ }^{7}$, Colleen Woods ${ }^{8}$, William R. Schief (i) ${ }^{5}$, Daniel Emerling ${ }^{4}$, C. Richter King (i) ${ }^{8}$ \& Jean-Philippe Julien (i) ${ }^{1,2,9}$

Transmission-blocking vaccines have the potential to be key contributors to malaria elimination. Such vaccines elicit antibodies that inhibit parasites during their development in Anopheles mosquitoes, thus breaking the cycle of transmission. To date, characterization of humoral responses to Plasmodium falciparum transmission-blocking vaccine candidate Pfs 25 has largely been conducted in pre-clinical models. Here, we present molecular analyses of human antibody responses generated in a clinical trial evaluating Pfs25 vaccination. From a collection of monoclonal antibodies with transmission-blocking activity, we identify the most potent transmission-blocking antibody yet described against Pfs25; 2544. The interactions of 2544 and three other antibodies with Pfs25 are analyzed by crystallography to understand structural requirements for elicitation of human transmission-blocking responses. Our analyses provide insights into Pfs25 immunogenicity and epitope potency, and detail an affinity maturation pathway for a potent transmission-blocking antibody in humans. Our findings can be employed to guide the design of improved malaria transmission-blocking vaccines.

\footnotetext{
${ }^{1}$ Program in Molecular Medicine, The Hospital for Sick Children Research Institute, 686 Bay Street, Toronto, ON M5G 0A4, Canada. ${ }^{2}$ Department of Biochemistry, University of Toronto, 1 King's College Circle, Toronto, ON M5S 1A8, Canada. ${ }^{3}$ Laboratory of Malaria and Vector Research, National Institute of Allergy and Infectious Diseases, National Institutes of Health, 12735 Twinbrook Parkway, Rockville, MD 20852, USA. ${ }^{4}$ Atreca, 500 Saginaw Drive, Redwood City, CA 94063-4750, USA. ${ }^{5}$ Department of Immunology and Microbial Science, The Scripps Research Institute, La Jolla, CA 92037, USA. ${ }^{6}$ Gene Therapy Program \& Orphan Disease Center, Perelman School of Medicine, The University of Pennsylvania, Philadelphia, PA 19104, USA. ${ }^{7}$ Fraunhofer USA Center for Molecular Biotechnology CMB, 9 Innovation Way, Newark, DE 19711, USA. ${ }^{8}$ PATH's Malaria Vaccine Initiative, 455 Massachusetts Avenue NW Suite 1000, Washington, DC 20001, USA. ${ }^{9}$ Department of Immunology, University of Toronto, 1 King's College Circle, Toronto, ON M5S 1A8, Canada. Correspondence and requests for materials should be addressed to J.-P.J. (email: jean-philippe.julien@sickkids.ca)
} 
$\mathrm{M}$ alaria remains a critical problem for human global health, exemplified by the estimated 219 million cases and approximately 435,000 deaths in $2017^{1}$. More than $90 \%$ of deaths related to malaria infection occur in Africa, where the parasite Plasmodium falciparum is responsible for $>99 \%$ of clinical cases ${ }^{1}$. P. falciparum is therefore the primary target of malarial prevention strategies. Despite the severe morbidity and mortality associated with the disease, a highly efficacious and durable vaccine has yet to be developed. A major obstacle to designing a malaria vaccine is the complex $P$. falciparum lifecycle, which occurs in both a human host and a female Anopheles mosquito vector ${ }^{2-4}$. As the proteomic expression profile of the parasite changes dramatically throughout its development, the identity of antigens displayed to the immune system varies greatly, and hence a combination of vaccination approaches that target its multiple life stages may be ideal ${ }^{2,5,6}$. The most advanced vaccine for malaria is the RTS,S/AS01 subunit vaccine approved for use in pilot implementations in parts of Ghana, Kenya, and Malawi $^{7,8}$. RTS,S is designed to elicit antibodies and T cells that block infection of the human liver following a mosquito bite. A complement to this strategy is to block infection at a different stage of $P$. falciparum development, the sexual stage in the mosquito, to prevent subsequent transmission of malaria-causing parasites to humans ${ }^{9-11}$. Vaccines designed to induce antibodies that function in the mosquito to prevent transmission to humans have been termed transmission-blocking vaccines (TBVs).

One of the most attractive TBV targets is Pfs25, a glycophosphotidylinositol-linked protein expressed on the surface of ookinetes, which develop from the fertilized zygote ${ }^{12,13}$. Pfs25 is important for ookinete survival in the protease-rich mosquito midgut, and Pfs 25 assists in the invasion of the midgut epithelium and maturation of the oocyst ${ }^{14,15}$. Pfs 25 exhibits minimal

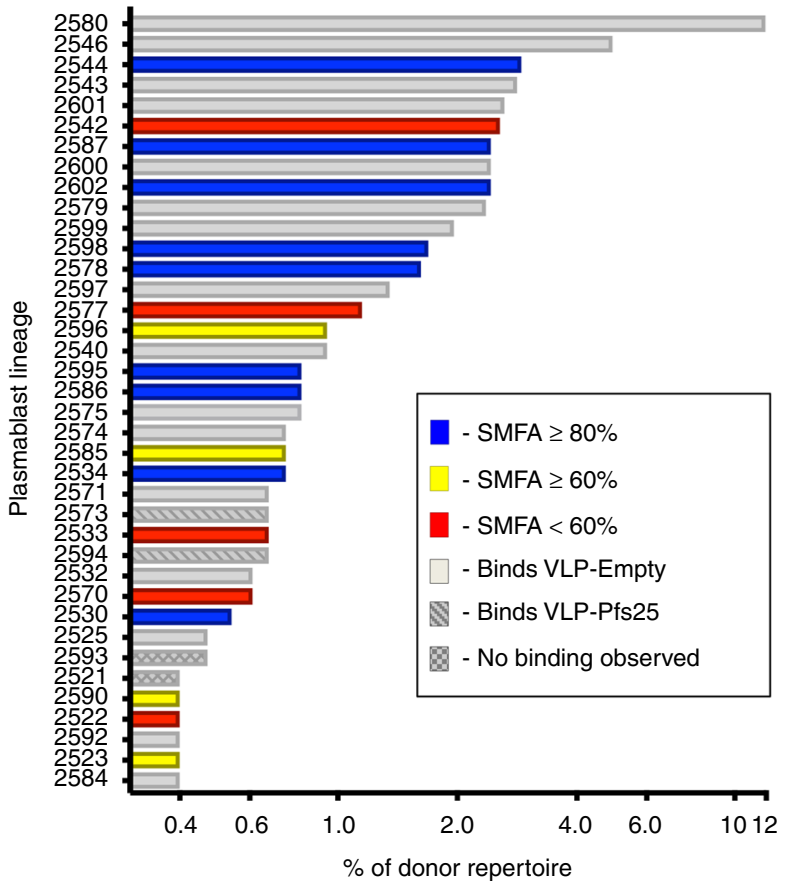

Fig. 1 Plasmablast responses of a human donor to Pfs25-VLP immunization. Rows correspond to different antibody lineages. Labels on the left correspond to a representative lineage member that was purified, expressed, and tested for binding activity by ELISA with Pfs 25 monomer, Pfs25-VLP (Pfs25+VLP) or VLP-empty (VLP alone with no antigen). VLPempty refers to only the virus-like particle that displays the antigen, as previously described ${ }^{31}$. Antibodies that bound to the Pfs 25 monomer were further tested by SMFA at $100 \mu \mathrm{g} / \mathrm{mL}$ sequence variation between isolates, presumably not only due to functional constraints but also likely due to the fact that Pfs 25 is expressed solely inside the mosquito and does not undergo the selective pressures of the human immune system ${ }^{16,17}$. When present in a blood meal, anti-Pfs 25 antibodies significantly inhibit the development of oocysts in an in vivo standard membrane feeding assay (SMFA) $12,18,19$. Together, these data suggest that, if anti-Pfs 25 antibodies are present in humans before a mosquito feeds on parasite-infected blood, $P$. falciparum development and onward transmission can be inhibited.

The main challenge in developing effective TBVs is to elicit highly potent antibodies at sufficient titers for durable transmission-blocking activity. To increase the magnitude of humoral responses, multimerization of Pfs 25 and its combination with different adjuvants have been explored. In comparison to soluble monomeric protein, Pfs 25 has been shown to be substantially more immunogenic when presented in a multimeric format across different particle platforms, including liposomes and viral particles ${ }^{20-22}$. Similarly, an increase in anti-Pfs 25 titers and functional antibody responses has been observed when Pfs 25 is conjugated with bacterial ExoProtein $\mathrm{A}$ in animal models ${ }^{23}$. Limited clinical testing of Pfs 25 vaccine candidates have been conducted to date, and in those cases only modest levels of functional transmission-blocking activity was observed ${ }^{24-26}$. Until recently, there has been very little information available to understand the level of functional activity of induced human antibody responses. To begin to bridge this gap, we reported on the structure and function of monoclonal antibodies (mAbs) against Pfs25 elicited by a plant-produced Pfs 25 displayed on alfalfa mosaic virus-like proteins (Pfs25-VLP) in Kymice, where the murine immunoglobulin (Ig) gene repertoire is replaced with that of humans ${ }^{27,28}$. Two distinct immunogenic sites were defined on Pfs25, with the most potent antibodies being directed to the epitope competing with the murine antibody 4B7, a wellcharacterized and potent $\mathrm{mAb}$ to $\mathrm{Pfs} 25^{27,29}$.

Here we explore the structure and function of transmissionblocking antibodies elicited in a human vaccinated with Pfs25$\mathrm{VLP}^{30}$. We determine the co-crystal structures of four human antibodies bound to Pfs25, and describe three novel protective epitopes on Pfs25, including the most potent $\mathrm{mAb}$ yet described from any organism. Studies of the plasmablast lineage from which this potent transmission-blocking antibody derives reveal the evolutionary steps required for potent parasite inhibition. These insights into the maturation of potent transmission-blocking antibodies in a human will help guide the development of increasingly effective biomedical interventions against malaria.

\section{Results}

Pfs25-VLP vaccination elicits a diverse plasmablast response. A total of 44 healthy human subjects were vaccinated in a doseescalation study with the plant-produced Pfs 25 -VLP adjuvanted in Alhydrogel ${ }^{\circledR 30,31}$. One of the individuals with the highest transmission-reducing activity (TRA) determined by SMFA was chosen for further cellular characterization ${ }^{30}$ (Supplementary Fig. 1). Plasmablasts were isolated and sequenced to obtain paired heavy- and light-chain genes as previously reported ${ }^{32,33}$. Representative mAbs from each of the most expanded 38 blood plasmablast lineages were expressed and assessed by enzyme-linked immunosorbent assay (ELISA) for binding to Pfs25-VLP, recombinant Pfs25, and VLP alone and classified according to their binding preference (Fig. 1 and Supplementary Fig. 2). Of these mAbs, 18 specifically bound the Pfs 25 monomer, two bound only Pfs25-VLP, 16 bound the VLP alone, and two did not bind any probes. Anti-Pfs $25 \mathrm{mAbs}$ that exclusively bound the Pfs 25 monomer were tested in SMFA to determine their TRA at a 
a

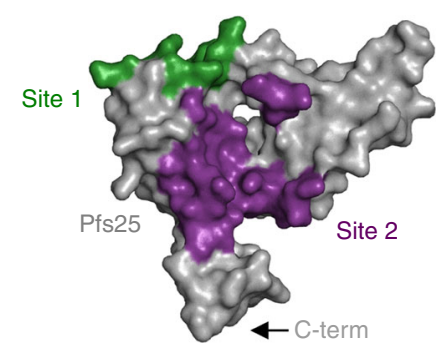

b

\begin{tabular}{|c|c|c|c|c|c|}
\hline \multirow[t]{2}{*}{ b } & \multicolumn{4}{|c|}{ Site 1} & \multirow{2}{*}{$\frac{\text { Site } 2}{1260}$} \\
\hline & 4B7 & 1269 & 1276 & 1266 & \\
\hline 30 & 100 & 76 & 47 & 51 & 82 \\
\hline 34 & 6 & 0 & 28 & 0 & 53 \\
\hline 544 & 1 & 35 & 56 & 3 & 70 \\
\hline 590 & 20 & 13 & 62 & 5 & 72 \\
\hline 502 & 14 & 0 & 15 & 60 & 80 \\
\hline 598 & 98 & 81 & 62 & 21 & 72 \\
\hline 23 & 90 & 52 & 0 & 5 & 0 \\
\hline 533 & 100 & 76 & 24 & 26 & 3 \\
\hline 7 & 70 & 0 & 86 & 0 & 0 \\
\hline 86 & 83 & 69 & 89 & 0 & 0 \\
\hline 2585 & 61 & 93 & 81 & 24 & 38 \\
\hline 2 & 71 & 81 & 59 & 6 & 7 \\
\hline & 74 & 50 & 59 & 6 & 7 \\
\hline 2 & 78 & 71 & 84 & 74 & 0 \\
\hline 2 & 97 & 85 & 56 & 63 & 10 \\
\hline
\end{tabular}

Fig. 2 Epitope binning of human anti-Pfs25 antibodies. a Surface representation of Pfs 25 showing the two previously characterized epitopes: Site 1 (1269, green) and Site 2 (1260, purple), and the C-terminus (C-Term) where Pfs 25 was fused to the VLP. b Previously characterized Fabs are listed along the top and are the primary Fabs in the BLI competition assay. Secondary (competing) human Fabs are to the left. Reported scores are a percentage of total potential binding of that antibody, and therefore higher numbers (blue) display low amounts of competition, while low numbers (red) exhibit greater competition. Any experiment with $>100 \%$ binding was given a score of 100 , while negative values were given a score of 0 . Human antibodies that fit previously defined epitopes Site 1 and Site 2 are outlined in green and purple, respectively. Potential novel epitope bins are highlighted by a dashed-line box

concentration of $100 \mu \mathrm{g} / \mathrm{mL}$. Nine of these mAbs (2530, 2534, $2544,2578,2586,2587,2595,2598$, and 2602) demonstrated $>80 \%$ reduction in oocyst density at $100 \mu \mathrm{g} / \mathrm{mL}$ concentration, while the other $9 \mathrm{mAbs}$ exhibited inhibition $<80 \%$ at this concentration (Fig. 1). The most expanded Pfs25-reactive plasmablast lineage was that of $\mathrm{mAb} 2544$ and represented approximately $3 \%$ of the total repertoire.

SMFA-active human mAbs bind a range of epitopes on Pfs25. Two distinct immunogenic sites on Pfs 25 had previously been defined structurally by characterizing mAbs derived from Pfs25VLP immunization in Kymice ${ }^{27}$. Here we used biolayer interferometry (BLI) to conduct competitive binding experiments between human-derived Fabs and Kymab Fab 1260 (prototypic
Site 2 epitope); Kymab Fabs 1266, 1269; and 1276 (prototypic Site 1 epitopes); and the well-characterized murine 4B7 Fab (also Site 1 epitope) (Fig. 2). Of the $18 \mathrm{mAbs}$ predicted by ELISA to specifically interact with Pfs25, 1 did not express in sufficient quantities as a Fab for analysis, and 2 did not bind Pfs 25 with detectable affinity via BLI. Of the 15 Fabs tested, 5 Fabs (2534, $2544,2590,2598$, and 2602) competed specifically with known antibodies directed to Site 1 or its sub-sites, and 2 Fabs (2570 and 2587) competed specifically with the Site 2-targeted antibody, corroborating the previous delineation of Pfs 25 immunogenicity $^{27}$. Interestingly, seven Fabs (2523, 2533, 2578, 2585, 2586, 2595, and 2596) competed with known antibodies from both Sites 1 and 2, suggesting that these sites may not be completely disparate but linked in one large immunogenic face, here termed the "bridging" epitope. Notably, mAb 2530 did not compete clearly with any previously defined site, suggesting mAb 2530 may bind a novel epitope on Pfs25, designated Site 3. The binding affinities of these Fabs to recombinant Pfs 25 ranged between 1.7 and $181 \mathrm{nM}$, and no clear association was observed between binding strength and which epitope was targeted (Supplementary Fig. 3). mAbs 2530, 2544, 2586, and 2587 have amongst the highest affinities to recombinant $\mathrm{Pfs} 25$ within their respective epitope bins (Fig. 3 and Supplementary Fig. 3) and also show $>80 \%$ oocyst reduction at $100 \mu \mathrm{g} / \mathrm{mL}$ in SMFA.

Crystal structures delineate novel Pfs25 epitopes. We next sought to delineate the epitopes of human mAbs, focusing primarily on specificities that had not yet been structurally characterized. Complexes of Fab 2530-Pfs25, Fab 2544-Pfs25, Fab 2586-Pfs25, and Fab 2587-Pfs 25 were crystallized and diffracted to 2.0, 2.9. 3.1, and $3.1 \AA$ resolution, respectively (Fig. 3, Supplementary Fig. 4, and Table 1). Each of the four structures corroborated the competition data, revealing novel epitopes and extended immunogenic faces beyond the previously distinct Sites 1 and 2 characterized for antibody recognition by SMFA-active antibodies elicited in Kymice ${ }^{27}$.

2586 binds directly in between Sites 1 and 2, with HCDR3 and $\kappa \mathrm{CDR} 3$ each overlapping a portion of both sites. 2586 has an epitope on Pfs 25 nearly identical to 2587 , despite a slightly different competition profile (Supplementary Fig. 5). This difference is explained by a slightly different angle of approach, resulting in the $2586 \kappa C D R 3$ partially impeding access to Site 1 . 2587 was identified as a Site 2-directed human Fab due to its strong competition with 1260. However, the crystal structure supports its categorization as a bridging antibody, also helping to explain its partial competition with Site 1-directed 1276. Notably, the only other Site 2-directed Fab, 2570, demonstrated poor TRA, even at a high concentration of $100 \mu \mathrm{g} / \mathrm{mL}$, confirming the poor potency previously associated with antibodies targeting this site ${ }^{27}$. 2530 binds almost exclusively to the Pfs25 EGF2 domain, with only few van der Waals interactions to EGF1, helping to explain its lack of clear competition with any of the previously defined mAbs (Fig. 3). This places the 2530 epitope (Site 3) on Pfs25 nearly opposite to those of 2586 and 2587. 2544 binds near the canonical Site 1 epitope but on the opposing side of the 4B7 loop when compared to the previously defined mAb 1269 epitope (Fig. 3). This shift from the prototypical Site 1-binding site puts 2544 nearly $180^{\circ}$ across from Site 2, an epitope not previously described. A consequence of all structural epitope mapping conducted to date is to indicate that most of the Pfs 25 surface is accessible as displayed on the VLP and elicits B cell responses after vaccination in humans (Supplementary Fig. 6).

2544 shows the most potent TRA. To determine the TRA of these antibodies against new epitope regions on Pfs 25 in more 
a

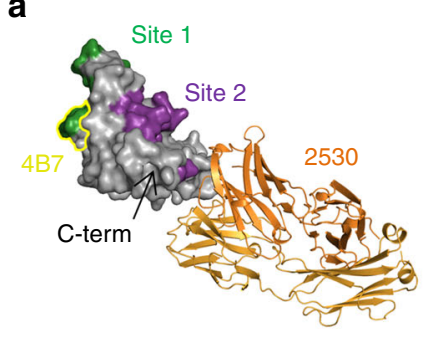

b

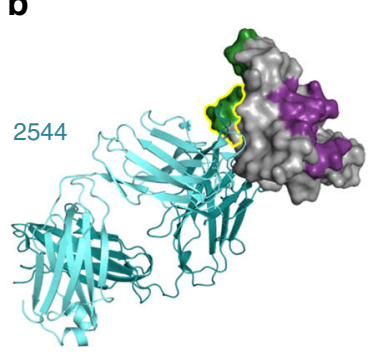

C

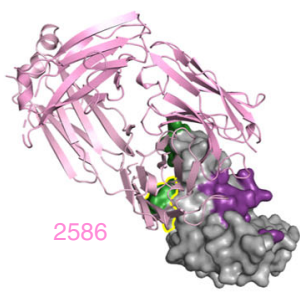

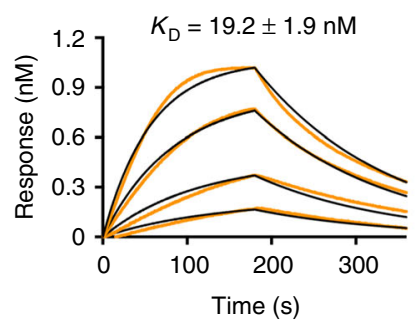
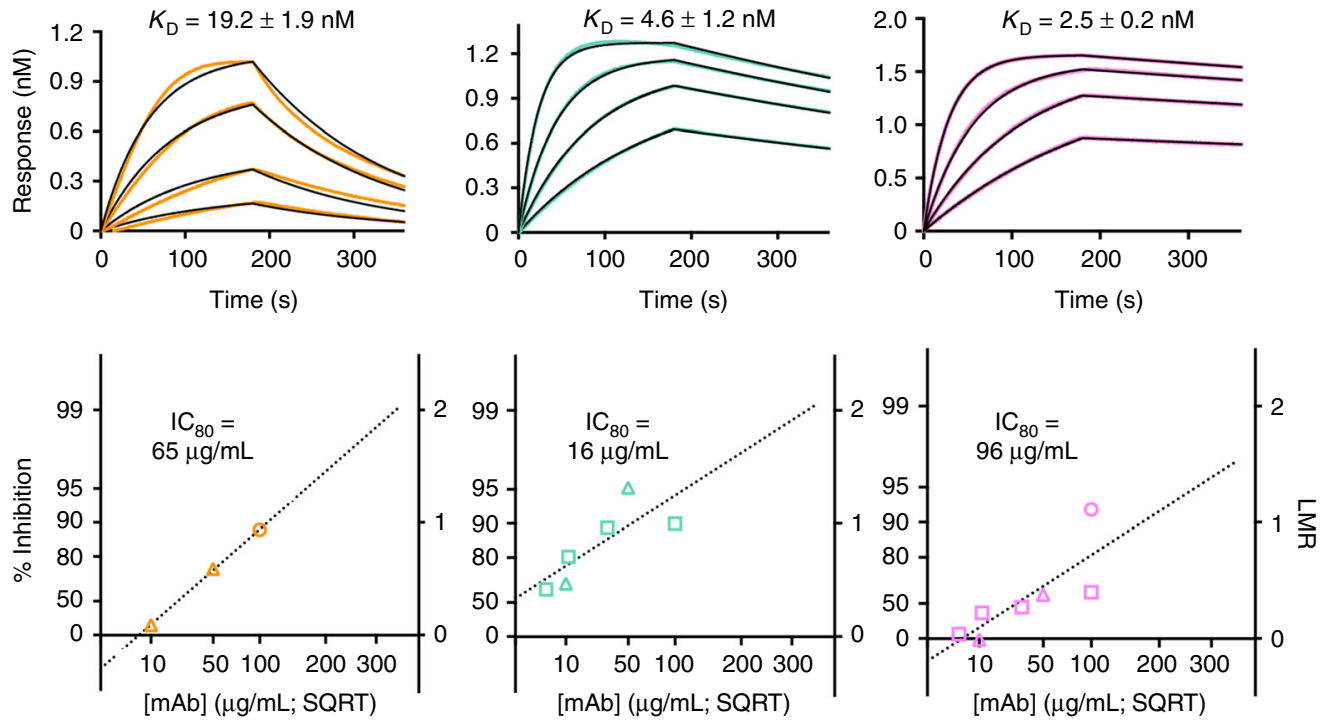

d

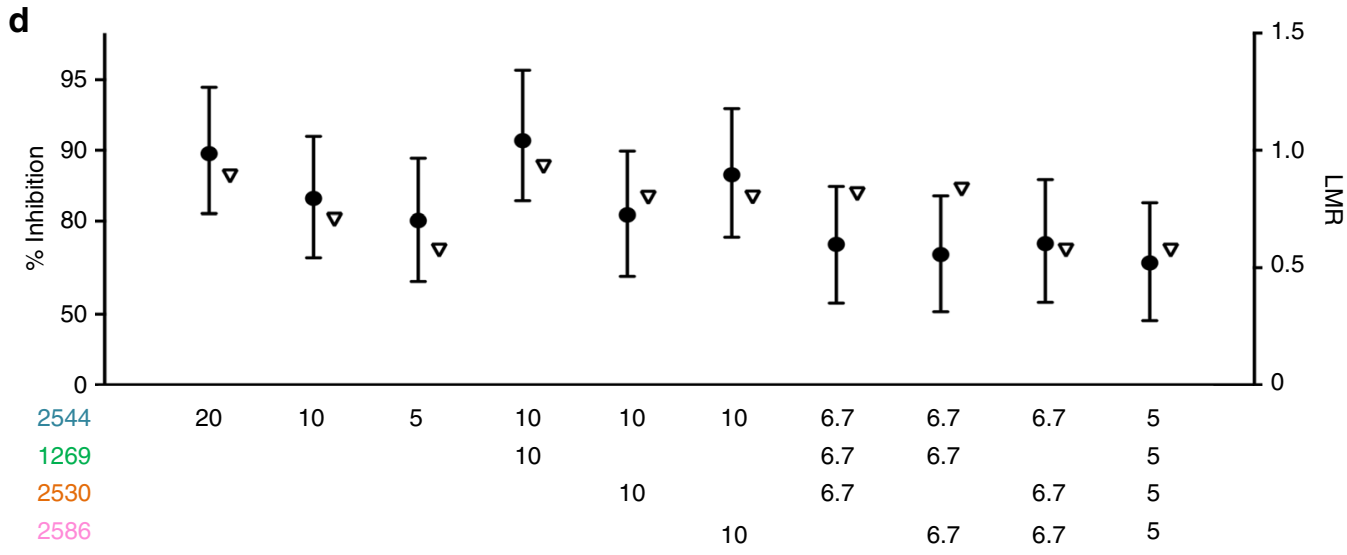

$[\mathrm{mAb}](\mu \mathrm{g} / \mathrm{mL})$

Fig. 3 Structure-function characterization of human antibodies against Pfs25. Top: crystal structure of Pfs 25 in complex with 2530 Fab (a, orange), 2544 Fab (b, teal), and 2586 Fab (c, pink). Site 1 (green), Site 2 (purple), the C terminus, and the 4B7 loop (yellow outline) are denoted in a. Middle: representative kinetic curves of $2530 \mathrm{Fab}$ (a), $2544 \mathrm{Fab}$ (b), and $2586 \mathrm{Fab}$ (c) binding to Pfs25, as measured by BLI, showing association and dissociation when Pfs 25 is immobilized on Ni-NTA biosensors and associated with serially diluted Fab (500-15.6 nM concentrations). The $K_{\mathrm{D}}$ is indicated and derives from three replicates with error defined as standard deviation. Bottom: SMFA of 2530 lgG (a), 2544 lgG (b), and 2586 lgG (c) at various concentrations across two feeds. Percentage of inhibition is plotted along the left $y$-axis, log of mean oocyst reduction (LMR) is plotted along the right $y$-axis. Shapes represent different SMFA feeds, and reported $\mathrm{IC}_{80}$ is calculated from the plotted line of best fit. $\mathbf{d}$ Combinatorial SMFA, plotted as above. Concentrations of the $\mathrm{mAbs}$ used are depicted along $x$-axis, and all combinations tested were at a cumulative mAb concentration of $20 \mu \mathrm{g} / \mathrm{mL}$. Circles depict the best estimate of SMFA inhibition for each mAb from two feeding experiments, while upside-down triangles refer to expected inhibition. For combinatorial SMFA, expected inhibition was calculated using the Bliss independence model. Error bars are $95 \%$ confidence intervals

detail, IgGs were tested in SMFA at multiple concentrations between 5 and $100 \mu \mathrm{g} / \mathrm{mL}$ across multiple feeds, and $\mathrm{IC}_{80}$ values were calculated. The $\mathrm{IC}_{80}$ values and their $95 \%$ confidence intervals (CIs) for 2530, 2544, and 2586 were determined to be 65
[95\% CI, 53-82]), 16 [95\% CI, 2-41]), and 96 [95\% CI, 57-145]) $\mu \mathrm{g} / \mathrm{mL}$, respectively (Fig. 3). Notably, 2544 has significantly higher TRA than any other mAb directed against Pfs 25 yet reported, including the previously described 1269 Site 1-directed 


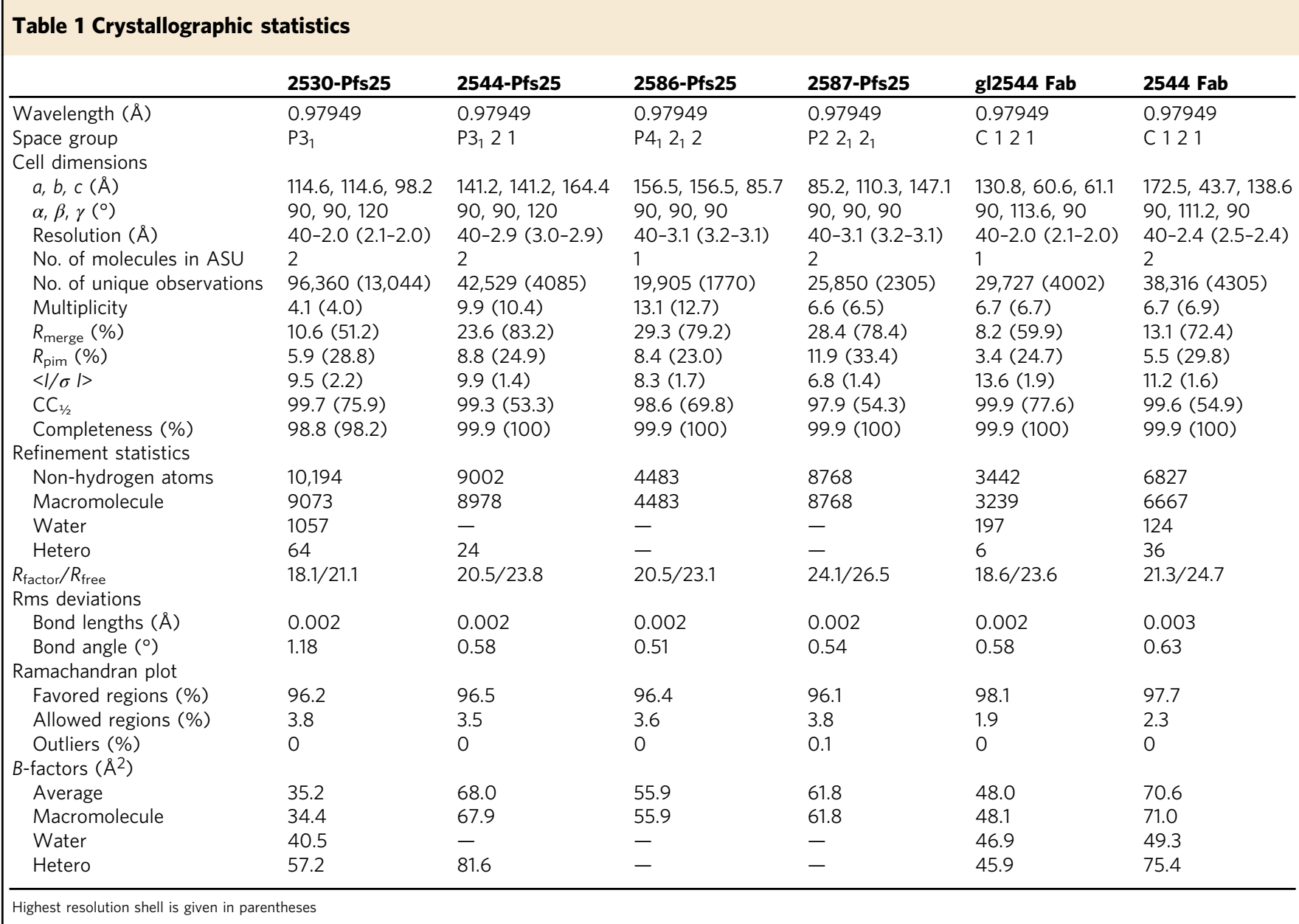

$\mathrm{mAb}$ derived from a Kymouse $\left(\mathrm{IC}_{80}=63 \mu \mathrm{g} / \mathrm{mL} \quad[95 \%\right.$ $\mathrm{CI}, 53-75])$ and the murine $4 \mathrm{~B} 7\left(\mathrm{IC}_{80}=29 \mu \mathrm{g} / \mathrm{mL}\right.$ [95\% CI, 24-34] $)^{27}$.

Our structural and competition analyses suggested that multiple antibodies could target Pfs 25 simultaneously. We confirmed by sequential binding using BLI that recombinant Pfs 25 can be bound concurrently by mAbs 2544, 1269, 2530, and 2586, with each Fab binding a different epitope (Supplementary Fig. 7). To determine how the TRA of human mAbs is affected when used in combination, SMFA experiments were conducted at a $20 \mu \mathrm{g} / \mathrm{mL}$ total antibody concentration, using either 2544 alone or in combination with up to three other mAbs. No synergy was observed, even when four separate epitopes were targeted simultaneously. Additive effects were observed when combining 1269 and 2544. However, owing to the substantially higher potency of 2544 compared to any other $\mathrm{mAb}$, we note that higher SMFA activity is obtained with higher doses $(20$ or $10 \mu \mathrm{g} / \mathrm{mL})$ of 2544 (Fig. 3d)

2544 forms extensive interactions with Pfs25. To gain understanding of the structural basis for the high potency of 2544 in SMFA, we carried out a detailed analysis of the interactions of 2544 with Pfs 25 . The 2544 Fab-Pfs 25 crystal structure revealed that the antibody buries $1005 \AA^{2}$ of surface area, and the majority of this interface is contributed by the heavy chain $\left(713 \AA^{2}\right)$, in comparison to the light chain $\left(292 \AA^{2}\right)$ (Fig. 4a). Correspondingly, the heavy chain forms 21 hydrogen bonds and one salt bridge with Pfs25, compared to four hydrogen bonds formed by the light chain. The HCDR3 of 2544 contributes the largest buried surface area (BSA) of any CDR $\left(368 \AA^{2}\right)$, and forms seven hydrogen bonds and one salt bridge, largely mediated by four tyrosines ( $\mathrm{Y}^{97}, \mathrm{Y}^{99}, \mathrm{Y}^{100 \mathrm{D}}, \mathrm{Y}^{100 \mathrm{~F}}$; Kabat numbering) that reach into a pocket of the EGF1 domain of Pfs25, immediately beside the 4B7 loop on EGF3 (Fig. 4b). Despite a slightly smaller BSA $\left(241 \AA^{2}\right)$, the HCDR2 forms 10 hydrogen bonds, five of which are mediated by $\mathrm{T}^{56}$ (Fig. 4c). All interactions formed by the light chain with Pfs 25 are mediated by $\kappa \mathrm{CDR} 1$ and $\kappa \mathrm{CDR} 3$, with four hydrogen bonds to the EGF4 domain of Pfs25 (Fig. 4d). $\kappa \mathrm{CDR} 2$ plays a structural role to help shape the 2544 paratope but does not interact with Pfs25.

Structural basis of 2544 affinity maturation. Sixteen amino acid exchanges separate 2544 from its inferred germline precursor (gl2544). Notably, gl2544 does not bind Pfs25 with appreciable affinity up to the $\mu \mathrm{M}$ range as a Fab and only with $2.5 \pm 1.1 \mu \mathrm{M}$ apparent binding avidity as an IgG; consequently, it fails to show any detectable inhibition in SMFA at a maximum tested concentration of $375 \mu \mathrm{g} / \mathrm{mL}$ (Supplementary Table 1). To understand the structural basis of affinity maturation, we solved the crystal structures of the unliganded $2544 \mathrm{Fab}$ and unliganded gl2544 Fab at 2.4 and $2.0 \AA$ resolution, respectively (Table 1 ). The crystal structure of unliganded 2544 showed high similarity to Pfs 25 bound 2544 (overall root mean square deviation $(\mathrm{RMSD})<0.5 \AA$ for the $\mathrm{F}_{\mathrm{v}}$ ). Comparison of the gl2544 structure with Pfs25-bound 2544 and unliganded 2544 revealed that the heavy chains are structurally similar, with a main-chain RMSD $<2.5 \AA$ throughout the variable region, with the exception of the HCDR3, which displays major differences (nearly $12 \AA$ in RMSD for certain residues) (Fig. 5a, top). The germline HCDR3 adopts an extended conformation in contrast to 2544 , which bends at the loop apex 


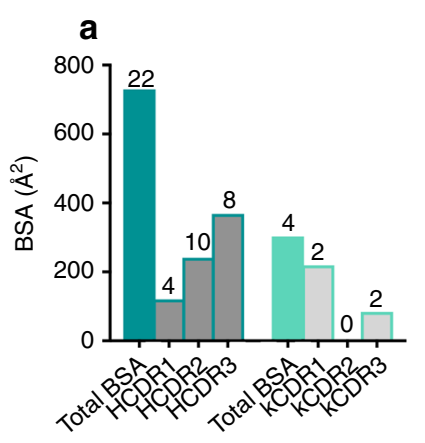

b

c
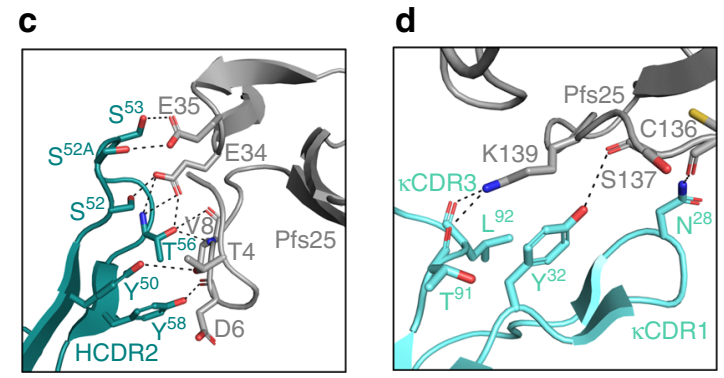

Fig. 4 Molecular definition of the interaction between mAb 2544 and Pfs25. a Plot of buried surface area (BSA) of the 2544-Pfs25 complex crystal structure. BSA is separated into total interaction of heavy chain (dark teal) and light chain (light teal). Chains are further delineated into the amount of BSA per CDR, as labeled. The total number of hydrogen bonds and salt bridges per chain and CDR is listed above each bar. $\mathbf{b}$ Interaction between the 2544 HCDR3 (dark teal) and Pfs25 (gray). Both proteins are displayed as cartoons, and the side chains or main chain of residues that form hydrogen bonds are displayed as sticks. Hydrogen bonds are denoted by black dashes. c Interactions between 2544 HCDR2 (dark teal) and Pfs25

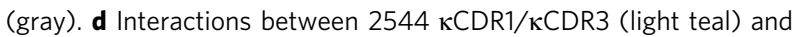
$\operatorname{Pfs} 25$ (gray)

(Fig. 5b). The extended conformation observed for the germline HCDR3 would be incompatible with binding to Pfs 25 without conformational rearrangement.

The light-chain arrangement varies considerably more between the gl2544 and 2544 antibodies (Fig. 5a, bottom). In contrast to the light-chain $\mathrm{N}$ terminus that shapes the binding site of 2544, the light-chain $\mathrm{N}$ terminus of gl2544 adopts an elongated conformation that points away from the paratope (Fig. 5c). Notably, this striking difference occurs despite there being any residue mutation in the light-chain $\mathrm{N}$ terminus resulting from affinity maturation. In addition, the apex of $\kappa \mathrm{CDR} 1$ is disordered in the germline structure, in contrast to 2544 where the $\kappa C D R 1$ is an ordered component of the binding site. From our comparative structural studies, we propose that there is an interconnectedness between the light-chain $\mathrm{N}$ terminus, $\kappa \mathrm{CDR} 1$ and $\kappa \mathrm{CDR} 3$ where somatic hypermutations in the $\kappa$ CDR3 result in stabilizing lightchain components in an optimal configuration for engaging Pfs25, from an otherwise flexible conformational ensemble in the germline antibody (Fig. 5c).

High-affinity binding achieved by minimal paratope mutations. We next investigated how the 16 somatic hypermutations in 2544 contributed to its activity. As a first step, all residues in 2544 were reverted to germline individually (except for $\mathrm{N}^{33}$, which would have introduced an N-linked glycosylation site not present in 2544) and binding to Pfs 25 was measured by BLI. Five mutations $\left(\mathrm{G}^{55} \mathrm{~S}\right.$ and $\mathrm{D}^{100 G} \mathrm{Y}$ in the heavy chain and $\mathrm{T}^{91} \mathrm{~A}, \mathrm{P}^{94} \mathrm{~T}$, and $\mathrm{F}^{95} \mathrm{P}$ in the light chain) appeared to be the most critical reversion mutations for Pfs 25 binding (Fig. 5d, e). Only two of these residues, $\mathrm{G}^{55}$ in the heavy chain and $\mathrm{P}^{94}$ in the light chain directly interact with Pfs25. In the unliganded gl2544 structure, $\mathrm{Y}^{100 \mathrm{G}}$ stabilizes an extended HCDR3 conformation, which would be unfavorable for Pfs 25 binding and likely explains the lower affinity for this reversion. The three mutations in the $\kappa$ CDR3 are near one another, and we propose these residues jointly act in stabilizing the light-chain paratope conformation to favorably interact with Pfs25.

Next, gl2544 that included the most critical 1, 3, or 5 mutations identified in the reversion experiments were expressed. The Fab construct with only one mutation from germline $\left(\kappa\right.$ chain $\mathrm{P}^{95} \mathrm{~F}$; referred to as gl2544+1), showed weak but detectable binding at a maximum concentration of $1.5 \mu \mathrm{M}$ (Fig. 5f). Since only the highest concentration showed detectable binding, a global fit to determine $K_{\mathrm{D}}$ was not possible. The 3 -mutation $\left(\kappa\right.$ chain $\mathrm{A}^{91} \mathrm{~T}$, $\mathrm{T}^{94} \mathrm{P}$, and $\mathrm{P}^{95} \mathrm{~F} ; \mathrm{gl} 2544+3$ ) and 5-mutation Fab constructs (heavy chain $\mathrm{S}^{55} \mathrm{G}$ and $\mathrm{Y}^{100 \mathrm{G}} \mathrm{D} ; \kappa$ chain $\mathrm{A}^{91} \mathrm{~T}, \mathrm{~T}^{94} \mathrm{P}$, and $\left.\mathrm{P}^{95} \mathrm{~F} ; \mathrm{gl} 2544+5\right)$ bound Pfs 25 with affinities of $2.0 \mu \mathrm{M}$ and $51.1 \mathrm{nM}$, respectively (Fig. 5f). Therefore, our data indicate that it takes only three mutations from the weakly binding gl2544 to confer low micromolar affinity for Pfs 25 , and only five mutations to achieve nanomolar binding. The 5-mutation antibody showed a weakerthan-2544 but significant SMFA activity of 78\% [95\% CI, 50-90]) at $375 \mu \mathrm{g} / \mathrm{mL}$ (Supplementary Table 1), indicating that a gain in binding affinity to this epitope is associated with inhibitory activity, as would be expected.

Antibodies across the $\mathbf{2 5 4 4}$ lineage show potent TRA. To better understand the development of potency for mAb 2544, we next examined the 2544 plasmablast lineage-the most expanded cellular and clonotypic Pfs25-specific lineage identified in this donor. An evolutionary phylogenetic tree of the 43 lineage members was generated through the analysis of variable domain sequences (Fig. 6a and Supplementary Fig. 8). Together, these antibodies differ from the inferred germline by 7-20 amino exchanges. Homology models were generated from all annotated antibody sequences using the 2544-Pfs 25 co-complex structure. Analysis of these homology models suggested that antibodies within the lineage ranged in binding affinity (intrinsic Molecular Operating Environment (MOE) scores ${ }^{34},-89$ to -104), BSA (1018 to $1172 \AA^{2}$ ), number of $\mathrm{H}$ bonds (10 to 19$), \Delta \mathrm{G}(-8.5$ to $-15.7 \mathrm{kcal} / \mathrm{mol}$ ), and stability (intrinsic MOE score ${ }^{34},-1945$ to -1979). Seven antibodies (30434, 30725, 5117, 5594, 6978, 8904, and 6012) were chosen for further characterization based on their ranking, selecting antibodies with high, intermediate, and low scores across these parameters (Table 2). Notably, 6012 possesses only seven amino acid exchanges from the inferred germline precursor, five of which are the most critical mutations for Pfs 25 binding identified in the germline reversion experiments described above. Binding kinetic experiments revealed a range of affinities to recombinant Pfs 25 for the selected lineage antibodies between 2.2 and $21.0 \mathrm{nM}$ (Table 2). As IgGs, all selected antibodies from the 2544 lineage reduced transmission in SMFA to a similar extent and within the confidence uncertainty of the assay (Fig. $6 \mathrm{~b}$ and Table 2). These data indicate that a ten-fold difference in binding affinity to recombinant Pfs 25 in the $<21.0 \mathrm{nM}$ range does not significantly impact the activity of 2544-lineage antibodies within the limits of the SMFA. Furthermore, the data highlight that antibodies of the 2544 lineage retain potency even with relatively few somatic hypermutations, making this lineage an attractive target to preferentially elicit by vaccination.

\section{Discussion}

Our study provides the first detailed molecular analysis of the structures and potencies of human mAbs targeting Pfs25, which 
a
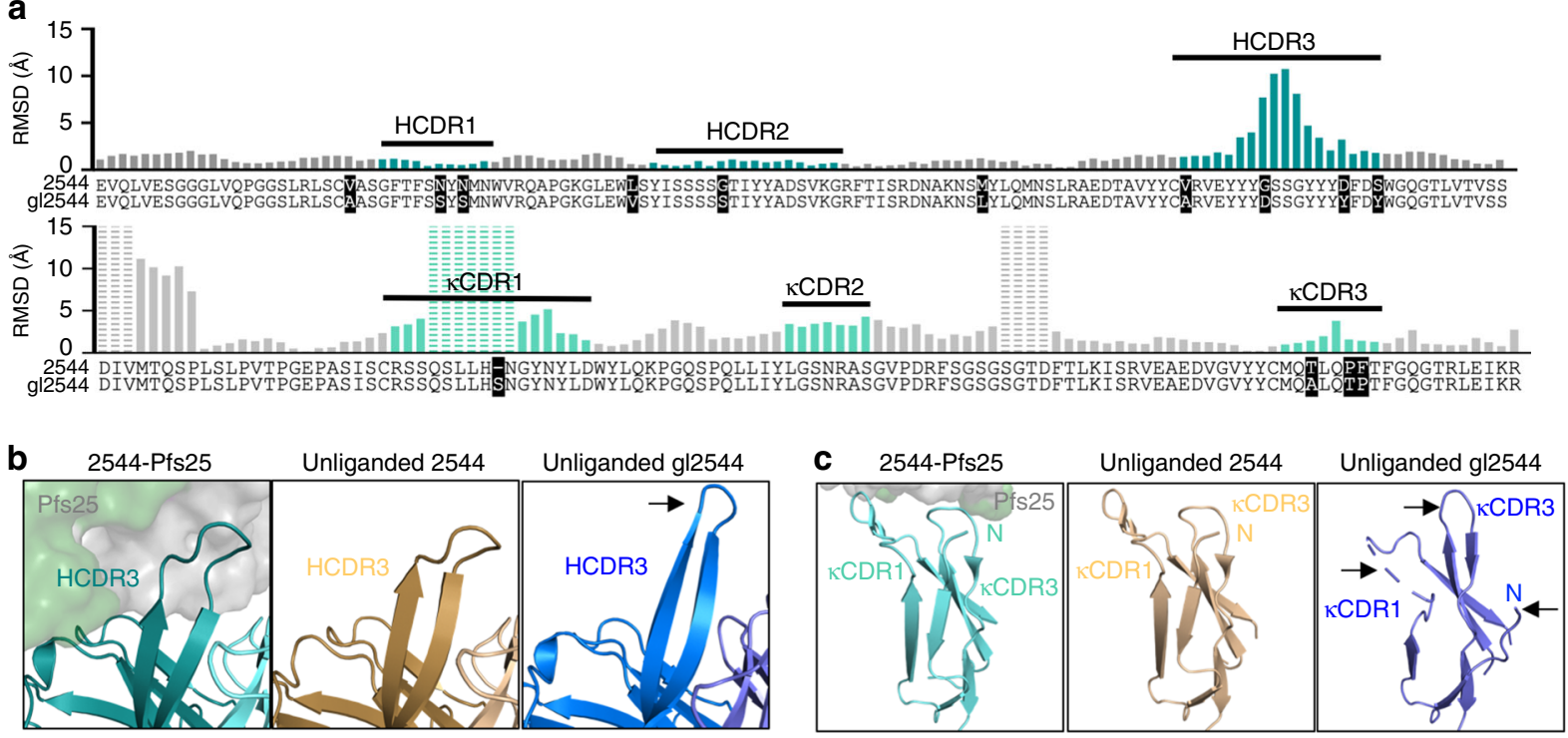

d

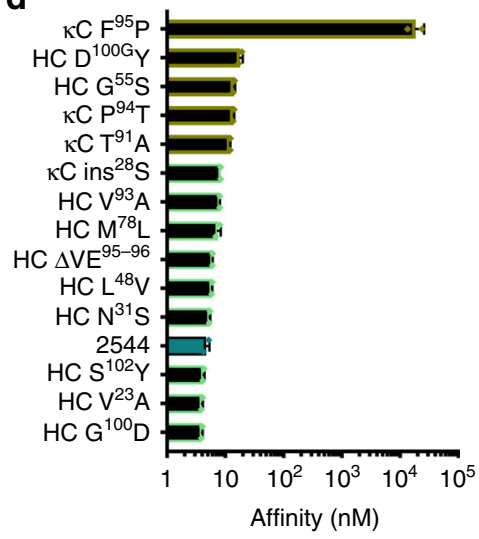

e

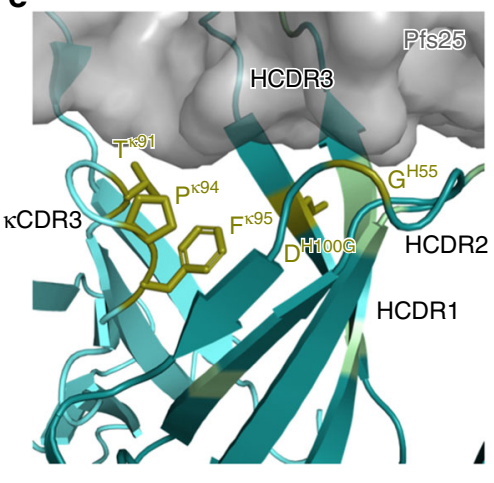

$\mathbf{f}$
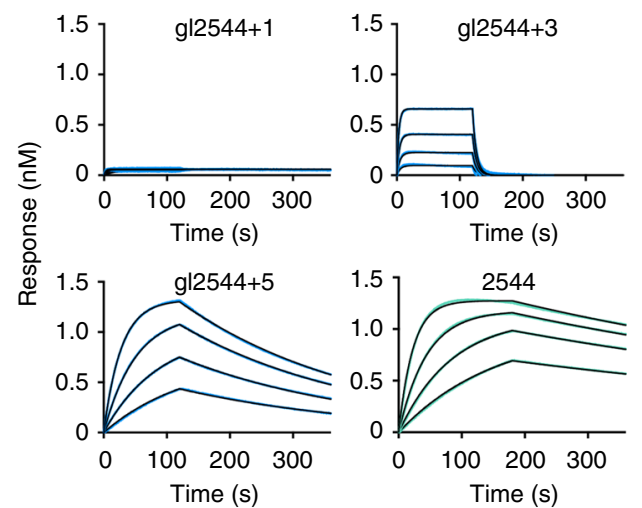

Fig. 5 Somatic hypermutations are required for 2544 high-affinity binding. a Alignment and RMSD plot between the variable regions of the heavy chains (top) and light chains (bottom) of Pfs25-bound 2544 and unliganded g12544. Somatic hypermutations from germline are highlighted in black. Gray bars of RMSD plot refer to framework regions, while heavy chain and light chain CDRs are denoted by dark and light teal bars, respectively. Dashed bars refer to residues that do not display density in at least one of the crystal structures. b Comparison of the HCDR3 of Pfs25-bound 2544 (teal, left), unliganded 2544 (beige, middle), and unliganded gl2544 (blue, right). Arrow indicates region of notable structural shift. c Comparison of the $\kappa$ CDR1 and $\kappa C D R 3$ of Pfs25bound 2544 (teal, left), unliganded 2544 (beige, middle), and unliganded gl2544 (blue, right). Arrows indicate regions of notable structural shift. d Affinities of 2544 germline-reversion mutations measured by BLI (immobilized Pfs 25 and mutant Fab analyte). The five reversions with the biggest impact on 2544 affinity are colored gold. Additional reversions are colored in pale green, and 2544 is colored in teal for comparison. Affinity is plotted as the mean of the measurements shown by solid diamonds, and the error is the standard deviation of the two kinetic measurements. e Position of the somatic hypermutations labeled in the $2544-\mathrm{Pfs} 25$ crystal structure according to the scheme in d. Pfs 25 is displayed in partial surface (gray), and 2544 is in teal. The five residues identified as most critical for binding are shown in gold sticks. $\mathbf{f}$ Representative kinetic curves for Fabs binding to Pfs 25 as measured by BLI (immobilized Pfs 25 and Fabs as analyte). The top left is gl2544 with the P95F mutation in the $\kappa$ chain ( $\mathrm{g} \mid 2544+1$ ), the top right is gl2544 with $A^{91} \mathrm{~T}, \mathrm{~T}^{94} \mathrm{P}$, and $\mathrm{P}^{95} \mathrm{~F}$ mutations in the $\kappa$ chain $(\mathrm{g} \mid 2544+3)$, the bottom left is gl2544 with $\mathrm{A}^{91} \mathrm{~T}, \mathrm{~T}^{94} \mathrm{P}$, and $\mathrm{P}^{95} \mathrm{~F}$ mutations in the $\kappa$ chain, and $\mathrm{S}^{55} \mathrm{G}$ and $\mathrm{Y}^{100 G} \mathrm{D}$ in the heavy chain ( $\left.\mathrm{g} \mid 2544+5\right)$, and the bottom right is 2544 . A maximal Fab concentration of $1.5 \mu \mathrm{M}$ and a minimum of $187.5 \mathrm{nM}$ for the gl2544 +1 and gl $2544+3$ mutants, and a concentration range of 500-62.5 nM for gl2544+5 and 2544 were used for kinetic determination experiments

were elicited during a phase I clinical trial with Pfs25-VLPs ${ }^{30}$. Previous molecular studies of ten mAbs derived from human Ig loci transgenic mice that received the same immunogen distinguished two binding sites on Pfs25, with Site 1 being considerably more immunogenic and targeted by more potent antibodies ${ }^{27}$. Notably, the extent to which humanized mice and other alternative antibody-generation platforms are reflective of human responses to vaccination remains unclear, reinforcing the need to characterize antibody responses from human vaccination. The human mAbs reported here bind a broader range of epitopes, including epitopes that bridge Sites 1 and 2, and another distinct epitope termed Site 3. Notably, the Site 3-directed mAb 2530 interacts with Pfs25 in a nearly identical manner as $2 \mathrm{~A} 8$, the canonical $\mathrm{mAb}$ to the $\mathrm{P} 25$ homolog in Plasmodium vivax ${ }^{35}$. A study in wild-type mice had suggested that this epitope was particularly immunogenic ${ }^{12}$, yet 2530 has one of the least clonally expanded lineages of inhibitory Pfs 25 antibodies and is the only Pfs25-directed mAb yet observed to this epitope. It remains unclear how the immunogenicity of Pfs 25 and Pvs 25 may differ, but our combined data from humanized mice and humans suggest that Pfs25 is primarily immunogenic around Site 1 (Fig. $7 \mathrm{a}$ ).

Our analysis also allows an assessment of the relationship between antibody-binding affinity and functional potency. Within each Pfs25 epitope bin, a range of affinities and molecular 

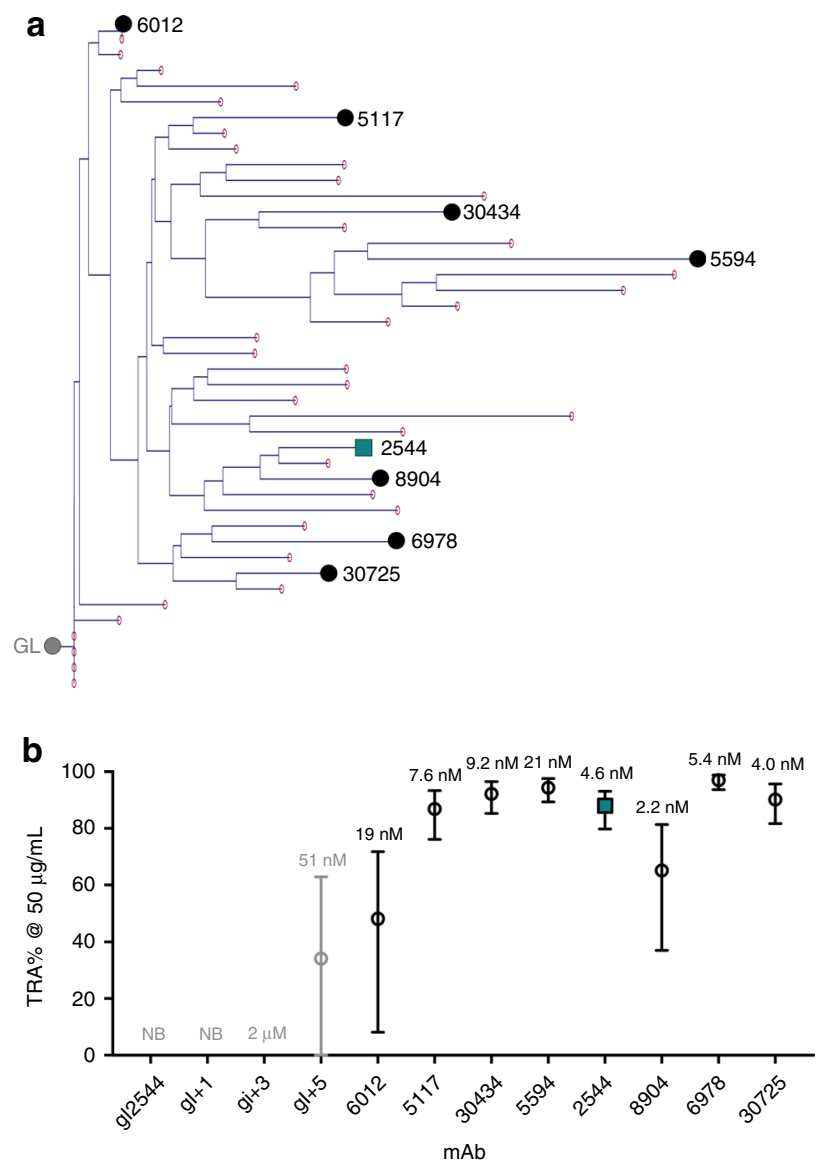

Fig. 6 The 2544 lineage shows potent SMFA activity. a Phylogenetic tree of the 2544 lineage. The gray, leftmost circle refers to the position of the inferred germline precursor. Red circles indicate sequenced members of the lineage; the length of blue lines represents the evolutionary distance from germline. Antibodies chosen for further characterization are denoted by a black circle and labeled. 2544 is displayed as a teal square. b Transmissionreducing activity (in percentage) for gl2544 (gray), germline mutants (gray), and 2544-lineage member mAbs (black) in SMFA at $50 \mu \mathrm{g} / \mathrm{mL} . K_{\mathrm{D}}$ for each Fab binding to recombinant Pfs 25 is denoted above its bar of SMFA activity. NB, no binding constant measured. Data are the result of two separate feeds, and the best-estimate (circles) and the $95 \% \mathrm{Cl}$ (error bars) of \%TRA values are shown

interactions are observed, and high-affinity binding to recombinant Pfs25 does not seem to be confined to any specific epitope (Supplementary Fig. 3). Interestingly, we did not detect any relationship between $\mathrm{mAb}$ binding kinetics to recombinant Pfs 25 and potency in SMFA. For example, mAb 2544 is significantly more potent than any other characterized anti-Pfs 25 antibody $\left(\mathrm{IC}_{80}=16 \mu \mathrm{g} / \mathrm{mL}\right)$, yet has a binding affinity to recombinant Pfs25 in the same low nanomolar range $\left(K_{\mathrm{D}}=4.6 \mathrm{nM}\right)$ as a variety of other mAbs with much lower potency $\left(1269: K_{\mathrm{D}}=\right.$ $3.7 \mathrm{nM}, \mathrm{IC}_{80}=63 \mu \mathrm{g} / \mathrm{mL} ; 1245: K_{\mathrm{D}}=31.0 \mathrm{nM}, \mathrm{IC}_{80}=263 \mu \mathrm{g} / \mathrm{mL}$, 2586: $K_{\mathrm{D}}=3.3 \mathrm{nM}, \mathrm{IC}_{80}=96 \mu \mathrm{g} / \mathrm{mL}$ ). In addition, molecular analysis of various members of the 2544 lineage demonstrates that differing amounts of hydrogen bonds or BSA or a log difference in binding affinity to recombinant Pfs25, do not significantly correlate with transmission-blocking activity for binding the same epitope within the detection limit. Therefore, we propose that potency is primarily determined by the epitope that is engaged (Fig. 7b and Supplementary Fig. 9) and is possibly related to the angle of approach. Indeed, 2544 recognizes the 4B7 loop at a shifted angle compared to other Site 1-directed mAbs of known structures (e.g., 1269, which has been shown to be of intermediate potency in SMFA, $\mathrm{IC}_{80}=63 \mu \mathrm{g} / \mathrm{mL}$, and murine $\left.4 \mathrm{~B} 7, \mathrm{IC}_{80}=29 \mu \mathrm{g} / \mathrm{mL}\right)^{27}$. Epitopes distant from Site 1 are associated with substantially lower SMFA activity $\left(1245, \mathrm{IC}_{80}=263\right.$ $\left.\mu \mathrm{g} / \mathrm{mL} ; 2586, \mathrm{IC}_{80}=96 \mu \mathrm{g} / \mathrm{mL}\right)$.

Combined, these data provide a guide for improved vaccine design based on immuno-focusing to mask immunogenic surfaces associated with poor mAb inhibitory activity and thereby direct B cell responses to sites associated with potent parasite inhibition. Such structure-based approaches have been successful in preferentially eliciting antibody responses to sites of vulnerability on the HIV-1 Env ${ }^{36,37}$, the Respiratory Syncytial Virus F protein $^{38}$, and the influenza hemagglutinin 39,40 , among other examples. Our findings that human-derived Pfs 25 polyclonal antibodies do not appear to act synergistically (Fig. 3d) strongly suggest that eliciting high titers of the most potent $\mathrm{mAb}$ lineages will be critical to improve vaccine potency. Furthermore, with antibodies of less potency than 2544 partially competing for its epitope, it may be important to design an immunogen that preferentially elicits the most effective mode of binding to Pfs25. Indeed, despite the 2544 lineage being the most expanded Pfs25specific plasmablast lineage in this vaccine, it only represented $3 \%$ of the total repertoire, with the rest evidently targeting unrelated antigens, the immunogen coat protein, or regions on Pfs 25 associated with lower inhibitory potency. Consequently, this unfocused polyclonal response resulted in the modest TRA detected in the clinical trial at the serum level (approximately $30 \%$ at $3.5 \mathrm{mg} / \mathrm{mL}^{30}$ ).

Our molecular work also shows how a potent human mAb can develop with as little as seven amino acid mutations (mAb 6012) from an inferred germline B cell precursor. This suggests that vaccines targeting this (or a similar) lineage may rapidly induce the development of protective antibodies, even when minimally mutated from their germline precursor. Benefits of such an approach would include a reduced immunization schedule. The very low affinity of gl 2544 for Pfs 25 suggests that efficient vaccine elicitation of 2544-like antibodies might benefit from the design of a slightly modified Pfs 25 antigen with appreciable affinity for 2544-like naive B cells. Such protein-engineering efforts are being extensively explored in HIV vaccine development, with technologies being developed to more efficiently engage specific germline precursors ${ }^{41-44}$. Combining next-generation immunogens with potent adjuvants that elicit high titers and durable responses will also be critical for vaccine efficacy, as preclinical studies have shown how different combinations of Pfs 25 and adjuvants can result in vastly different antibody responses ${ }^{45-47}$.

Finally, our identification of a highly potent human $\mathrm{mAb}$ against Pfs 25 offers the opportunity to test its efficacy in passive immunization trials, such as is currently being developed for other transmission-blocking targets, e.g., Pfs $48 / 45^{48}$. Such data would be critical to further validate Pfs 25 as a TBV target and would determine the circulating antibody concentrations required to block the transmission of the parasite, thereby guiding targets for future Pfs25-based vaccine evaluation. The fine mapping of epitopes and their associated potency derived in our studies will also provide new tools to probe immune responses in future experimental animal and human studies, such as determining the proportion of a polyclonal response to Pfs 25 that binds the most potent epitopes. Taken together, our data provide a molecular framework for understanding human antibody responses to Pfs 25 and for designing improved transmission-blocking biomedical interventions.

\section{Methods}

Identification and selection of Pfs25-specific mAb sequences. Our analysis focused on the B cell response of one participant who achieved high TRA (76.9\% at 
Table 2 Characterization of mAbs from the 2544 lineage

\begin{tabular}{|c|c|c|c|c|c|c|c|c|c|}
\hline $\mathbf{m A b}$ & $\begin{array}{l}\text { Mutations } \\
\text { from gl }\end{array}$ & $\begin{array}{l}\text { BSA } \\
\left(\AA^{2}\right)\end{array}$ & HB & SB & $\begin{array}{l}\Delta G+H B / S B \\
(\mathrm{kcal} / \mathrm{mol})\end{array}$ & $\begin{array}{l}\text { MOE } \\
\text { affinity }\end{array}$ & $\begin{array}{l}\text { MOE } \\
\text { stability }\end{array}$ & $K_{\mathrm{D}}(\mathrm{M})$ & $\begin{array}{l}\text { TRA (\%) at } 50 \mu \mathrm{g} / \mathrm{mL} \\
(95 \% \mathrm{CI})\end{array}$ \\
\hline $\mathrm{g} 12544$ & - & 1097 & 12 & 1 & -10.8 & -96.7 & -1950.3 & NB & $1(-75$ to 45$)$ \\
\hline 2544 & 15 & 1005 & 25 & 1 & -15.6 & -102.6 & -1967.3 & $4.6 \times 10^{-9} \pm 1.2 \times 10^{-9}$ & $88(80$ to 93$)$ \\
\hline 30434 & 19 & 1140 & 15 & 3 & -15.2 & -98.9 & -1977.6 & $9.2 \times 10^{-9} \pm 6.1 \times 10^{-10}$ & $92(85$ to 97$)$ \\
\hline 30725 & 14 & 1101 & 15 & 2 & -12.5 & -102.0 & -1973.4 & $4.0 \times 10^{-9} \pm 6.4 \times 10^{-10}$ & $90(82$ to 96$)$ \\
\hline 5117 & 17 & 1031 & 11 & 1 & -8.5 & -94.6 & -1978.5 & $8.7 \times 10^{-9} \pm 1.6 \times 10^{-9}$ & 87 (76 to 93 ) \\
\hline 5594 & 20 & 1096 & 11 & 0 & -10.4 & -95.2 & -1952.8 & $2.1 \times 10^{-8} \pm 3.5 \times 10^{-10}$ & 94 (89 to 98$)$ \\
\hline 6012 & 7 & 1084 & 15 & 2 & -12.6 & -95.4 & -1962.8 & $1.9 \times 10^{-8} \pm 3.7 \times 10^{-9}$ & $48(8$ to 72$)$ \\
\hline 6978 & 15 & 1128 & 18 & 4 & -15.7 & -95.0 & -1969.4 & $5.4 \times 10^{-9} \pm 8.6 \times 10^{-10}$ & 97 (94 to 99$)$ \\
\hline 8904 & 17 & 1128 & 19 & 1 & -12.4 & -101.5 & -1976.2 & $2.2 \times 10^{-9} \pm 7.5 \times 10^{-10}$ & 65 (37 to 81$)$ \\
\hline
\end{tabular}

In bold, in silico modeling

BSA buried surface area, HB hydrogen bonds, SB salt bridges, $\triangle G$ Gibbs free energy, MOE Molecular Operating Environment, NB no binding, TRA transmission reducing activity

a

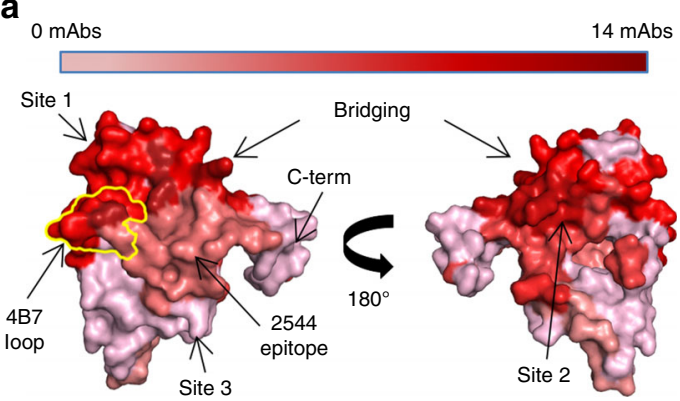

b

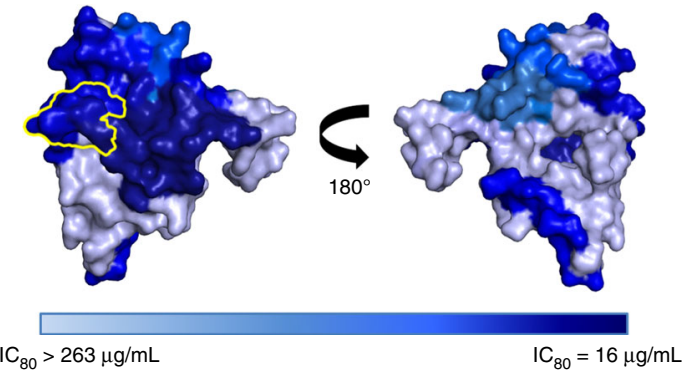

Fig. 7 Summary of Pfs 25 immunogenicity and mAb potency. a Binding sites on Pfs 25 of $\mathrm{mAbs}$ isolated from a human donor and previously isolated from Kymice ${ }^{27}$. Pfs 25 sites are delineated according to canonical binders for which crystal structures are known: 1269 for Site 1a, 2544 for Site 1b, 1260 for Site 2, 2586 for bridging, and 2530 for Site 3. Residues of Pfs 25 are colored according to the quantity of $m A b s$ expected to bind that residue from BLI competition experiments. The darker the red, the greater the immunogenicity on Pfs25. The loop on Pfs 25 recognized by murine 4B7 is highlighted in yellow. $\mathbf{b}$ Surface representation of potency for anti-Pfs 25 mAbs isolated from a human donor and Kymice ${ }^{27}$. Pfs 25 sites are delineated as in $\mathbf{a}$, and residues colored according to $\mathrm{mAb}$ potency $\left(\mathrm{IC}_{80}\right)$. If more than one $\mathrm{mAb}$ was present in an epitope bin, an average of the $\mathrm{IC}_{80} \mathrm{~S}$ was used. The darker the blue, the higher the potency of mAbs against this site in SMFA

$15 \mathrm{mg} / \mathrm{mL}$ ) in the phase I clinical trial study registered at http://www.ClinicalTrials. gov under reference identifier NCT02013687 $7^{30}$. Plasmablasts were isolated from peripheral blood mononuclear cells (PBMCs) using flow cytometry. PBMCs were stained with the following antibodies: anti-CD19 (clone HIB19, Brilliant Violet 421, BioLegend, cat \# 302234, $50 \mu \mathrm{g} / \mathrm{mL}, 1: 50$ ), anti-CD20 (2H7, PE-Cy7, BioLegend or L27, PerCP-Cy5.5, BD Bioscience, cat \# 302312, $100 \mu \mathrm{g} / \mathrm{mL}, 1: 100$ ), anti-CD27 (O323, Brilliant Violet 510, BioLegend, cat \# 302836, $100 \mu \mathrm{g} / \mathrm{mL}, 1: 50$ ), anti-CD38 (HIT2, Alexa647, BioLegend, cat \# 303514, $200 \mu \mathrm{g} / \mathrm{mL}, 1: 100$ ), anti-IgA (IS118E10, fluorescein isothiocyanate (FITC), Miltenyi Biotec, cat \# 130-093-071, $16.5 \mu \mathrm{g} / \mathrm{mL}, 1: 200$ ), anti-IgM (MHM-88, FITC, BioLegend, cat \# 314506,

$100 \mu \mathrm{g} / \mathrm{mL}, 1: 100$ ), anti-IgD (IA6-2, Alexa Fluor, BioLegend, cat \# 348216, $200 \mu \mathrm{g} /$ $\mathrm{mL}, 1: 100$ ), anti-CD3 (UCHT1, FITC, BioLegend, cat \# 300406, $200 \mu \mathrm{g} / \mathrm{mL}, 1: 100$ ), and anti-CD14 (HCD14, FITC, BioLegend, cat \# 325604, $400 \mu \mathrm{g} / \mathrm{mL}, 1: 100$ ). Flow cytometric analysis and cell sorting was performed on a BD FACSJazz using the BD FACS Software. Plasmablasts were isolated by gating for CD19+CD20low/CD27+CD38highIgA-IgM-IgD-CD3-CD14- cells. All paired chain antibody sequencing was carried out on IgG plasmablasts sorted into microtiter plates at one cell per well by fluorescence-activated cell sorter (FACS) and 1484 paired chain sequences were generated using Atreca's Immune Repertoire Capture ${ }^{\circledR}$ technology as previously described ${ }^{27}$. A total of thirty-eight paired IgG variable regions (CDRs and framework regions) were selected for direct recombinant expression from the most expanded plasmablast lineages identified from the donor sample. This approach biased the selection toward large expanded lineages.

Expression and purification of IgGs. Selected IgG sequences were expressed by Lake Pharma (Belmont, CA). Proteins were expressed using $0.1 \mathrm{~L}$ transient production in HEK293 cells (Thermo Fisher Scientific) and purified using protein A affinity chromatography. Purified proteins were $0.2 \mu \mathrm{m}$ sterile filtered, characterized for $>90 \%$ purity by CE-SDS, concentration and endotoxin $<100 \mathrm{EU} / \mathrm{mg}$ prior to vialing. Final buffer for all proteins contained $200 \mathrm{mM}$ HEPES, $100 \mathrm{mM} \mathrm{NaCl}$, and $50 \mathrm{mM} \mathrm{NaOAc}$, pH 7.0.

Expression and purification of Fabs. $\mathrm{V}_{\mathrm{L}}$ and $\mathrm{V}_{\mathrm{H}}$ regions were cloned into pcDNA3.4 expression vectors upstream of the human $\mathrm{C}_{\mathrm{H}}, \mathrm{C \kappa}$, or $\mathrm{C} \lambda$ regions. IgGs and Fabs were transiently expressed in HEK293F cells (Thermo Fisher Scientific) and purified using KappaSelect or LambdaSelect affinity (GE Healthcare), followed by cation exchange (MonoS, GE Healthcare) and size exclusion chromatography (Superdex 200 Increase 10/300 GL, GE Healthcare).

BLI binding studies. BLI (Octet RED96, FortéBio) experiments at $25^{\circ} \mathrm{C}$ were conducted to determine the epitope bins and binding kinetics of Pfs 25 and the human Fabs. Recombinant His-tagged Pfs25 purified from HEK293F cells (Thermo Fisher Scientific) through a 5-mL HisTrap FF column (GE Healthcare), followed by size exclusion chromatography (Superdex 200 Increase 10/300 GL, GE Healthcare ${ }^{27}$, was diluted into kinetics buffer (phosphate-buffered saline, $\mathrm{pH}$ 7.4, $0.01 \%(\mathrm{w} / \mathrm{v}$ ) bovine serum albumin, $0.002 \%(\mathrm{v} / \mathrm{v})$ Tween-20) at $10 \mu \mathrm{g} / \mathrm{mL}$ and immobilized onto Ni-NTA (NTA) biosensors (FortéBio). To determine epitope bins, baseline was gathered for $30 \mathrm{~s}$ before biosensors were dipped into wells containing the primary antibody $(\mathrm{Fab}, 10 \mu \mathrm{g} / \mathrm{mL}$ ) for $10 \mathrm{~min}$, followed by another $30 \mathrm{~s}$ baseline, and then dipped into wells containing a second antibody (Fab, $10 \mu \mathrm{g} /$ $\mathrm{mL}$ ) for $5 \mathrm{~min}$. For binding kinetics determination, His-tagged Pfs 25 was similarly diluted into kinetics buffer and immobilized onto Ni-NTA (NTA) biosensors (FortéBio). Following a 60-s baseline step, biosensors were dipped into wells containing twofold dilution series of Fab (affinity measurement) or IgG (apparent avidity measurement). Comparatively, IgG affinity was assessed by immobilizing the IgG onto anti-human Fc biosensors (FortéBio) and dipping into serially diluted concentrations of Pfs25. Sensors were then dipped back into kinetics buffer to monitor the dissociation rate. Competition and kinetics data were analyzed using the FortéBio's Data Analysis software 9.0, and kinetic curves were fitted to a 1:1 binding model using at least four concentrations. For experiments with several replicates, the mean kinetic constants reported are the result of two or more independent experiments, and associated error is standard deviation.

Co-crystallization and structure determination. A Pfs 25 construct with potential N-linked glycosylation sites at positions 91,144 , and 166 mutated to Gln was transiently expressed in HEK293F cells (Thermo Fisher Scientific) and purified via a 5-mL HisTrap FF column (GE Healthcare), followed by size exclusion 
chromatography (Superdex 200 Increase 10/300 GL, GE Healthcare), as previously described $^{27}$. Pfs 25 and human Fabs were mixed in a 2:1 molar ratio, and size exclusion chromatography was used to remove excess Pfs 25 . Fab-Pfs 25 complexes were concentrated to approximately $10 \mathrm{mg} / \mathrm{mL}$ and mixed 1:1 with mother liquor and set up in sitting-drop crystallization experiments. 2530-Pfs 25 crystals grew in $0.2 \mathrm{M}$ lithium sulfate, $0.1 \mathrm{M}$ Tris, $\mathrm{pH} 7.0$, and $2.0 \mathrm{M}$ ammonium sulfate and were cryoprotected with $15 \%(\mathrm{v} / \mathrm{v})$ ethylene glycol. 2544-Pfs 25 crystals were grown in $0.1 \mathrm{M}$ HEPES NaOH, pH 7.5, 20\% (w/v) PEG 4000, and 10\% (v/v) 2-propanol and cryoprotected with $10 \%(\mathrm{v} / \mathrm{v})$ glycerol. 2586-Pfs 25 crystals were grown in $0.2 \mathrm{M}$ lithium sulfate, $0.1 \mathrm{M}$ Tris-HCl, $\mathrm{pH} 8.5$, and 30\% (w/v) PEG 4000. 2587-Pfs 25 crystals were grown in $0.2 \mathrm{M}$ potassium thiocyanate, 20\% (w/v) PEG 3350, and cryoprotected in $15 \%(\mathrm{v} / \mathrm{v})$ ethylene glycol. Unliganded gl2544 was crystallized in $0.1 \mathrm{M}$ sodium acetate $\mathrm{pH} 4.5,25 \%$ (w/v) PEG 3350, and cryoprotected with $15 \%$ $(\mathrm{v} / \mathrm{v})$ glycerol. Unliganded 2544 crystals were grown in $0.1 \mathrm{M}$ sodium cacodylate $\mathrm{HCl}, \mathrm{pH} 6.5,1.0 \mathrm{M}$ sodium citrate tribasic, and cryoprotected with $15 \%(\mathrm{v} / \mathrm{v})$ glycerol. Data were collected at the 08ID-1 beamline at the Canadian Light Source (CLS), processed, and scaled using XDS ${ }^{49}$. Structures were determined by molecular replacement using Phaser and Pfs 25 as a search model ${ }^{27,50}$. Refinement of the structures was carried out using phenix.refine and model building iterations in Coot $^{51,52}$. SBGrid was used to access all software ${ }^{53}$.

Molecular modeling of $\mathbf{2 5 4 4}$ lineage. All models were generated from the 2544Pfs 25 complex crystal structure. This structure was prepared using QuickPrep in MOE, and models were generated through MOE's residue scanning function ${ }^{34}$. Predicted affinities between antibodies and the antigen were determined by model generation using MOE's scoring algorithms. Resulting homology models were further analyzed using the PDBePISA server ${ }^{54}$.

Standard membrane feeding assay. The ability of anti-Pfs 25 antibodies to reduce the development of $P$. falciparum NF54 strain oocysts in the mosquito midgut was evaluated by SMFA as described previously ${ }^{55}$. In brief, test antibodies at the indicated concentrations were mixed with $0.15-0.2 \%$ stage $\mathrm{V}$ gametocytemia and then fed to 3-6-day-old female Anopheles stephensi (initially provided by The Catholic University of the Netherlands). The mosquitoes were maintained for 8 days and then dissected to count the number of oocysts per midgut in 20 mosquitoes. The best estimate of \%TRA, the $95 \%$ CI, and significance of inhibition from single or multiple feeds were calculated as previously described using a zeroinflated negative binomial model ${ }^{56}$. The Bliss independence model, i.e., assuming $\mathrm{mAbs}$ act independently, was used to determine the theoretical additive effect.

Reporting summary. Further information on research design is available in the Nature Research Reporting Summary linked to this article.

\section{Data availability}

Crystallographic data has been deposited to the Protein Data Bank (PDB) under accession numbers $6 \mathrm{PHB}, 6 \mathrm{PHC}, 6 \mathrm{PHD}, 6 \mathrm{PHF}, 6 \mathrm{PHG}$, and $6 \mathrm{PHH}$. The authors declare that all other data supporting the findings of this study are available within the article and its Supplementary Information files or are available from the authors upon request.

\section{Received: 11 April 2019 Accepted: 14 August 2019}

Published online: 24 September 2019

\section{References}

1. WHO. WHO | World malaria report 2018. (WHO, 2018).

2. Florens, L. et al. A proteomic view of the Plasmodium falciparum life cycle. Nature 419, 520-526 (2002).

3. Gull, D. E. A. K. The Plasmodium cell cycle: facts and questions. Ann. Trop. Med. Parasitol. 92, 361-365 (1998)

4. Doerig, C., Chakrabarti, D., Kappes, B. \& Matthews, K. The cell cycle in protozoan parasites. Prog. Cell Cycle Res. 4, 163-183 (2000).

5. Saul, A. \& Fay, M. P. Human immunity and the design of multi-component, single target vaccines. PLoS ONE 2, e850 (2007).

6. Sherrard-Smith, E. et al. Synergy in anti-malarial pre-erythrocytic and transmission-blocking antibodies is achieved by reducing parasite density Elife 7, e35213 (2018).

7. RTS,S Clinical Trials Partnership. Efficacy and safety of RTS,S/AS01 malaria vaccine with or without a booster dose in infants and children in Africa: final results of a phase 3 , individually randomised, controlled trial. Lancet $\mathbf{3 8 6}$, 31-45 (2015).

8. RTS,S Clinical Trials Partnership et al. A phase 3 trial of RTS,S/AS01 malaria vaccine in African infants. N. Engl. J. Med. 367, 2284-2295 (2012).

9. De Zoysa, A. P. K., Herath, P. R. J., Abhayawardana, T. A., Padmalal, U. K. G. K. \& Mendis, K. N. Modulation of human malaria transmission by anti- gamete transmission blocking immunity. Trans. R. Soc. Trop. Med. Hyg. 82, 548-553 (1988).

10. Nunes, J. K. et al. Development of a transmission-blocking malaria vaccine: progress, challenges, and the path forward. Vaccine 32, 5531-5539 (2014).

11. Nikolaeva, D., Draper, S. J. \& Biswas, S. Toward the development of effective transmission-blocking vaccines for malaria. Expert Rev. Vaccin. 14, 653-680 (2015).

12. Stowers, A. W., Keister, D. B., Muratova, O. \& Kaslow, D. C. A region of Plasmodium falciparum antigen Pfs 25 that is the target of highly potent transmission-blocking antibodies. Infect. Immun. 68, 5530-5538 (2000).

13. Kaslow, D. C. et al. A vaccine candidate from the sexual stage of human malaria that contains EGF-like domains. Nature 333, 74-76 (1988).

14. Tomas, A. M. et al. P25 and P28 proteins of the malaria ookinete surface have multiple and partially redundant functions. EMBO J. 20, 3975-3983 (2001).

15. Baton, L. A. \& Ranford-Cartwright, L. C. Do malaria ookinete surface proteins P25 and P28 mediate parasite entry into mosquito midgut epithelial cells? Malar. J. 4, 15 (2005).

16. Lee, S. M. et al. Assessment of Pfs25 expressed from multiple soluble expression platforms for use as transmission-blocking vaccine candidates. Malar. J. 15, 405 (2016)

17. Kaslow, D. C., Quakyi, I. A. \& Keister, D. B. Minimal variation in a vaccine candidate from the sexual stage of Plasmodium falciparum. Mol. Biochem. Parasitol. 32, 101-103 (1989).

18. Kaslow, D. C. Immunogenicity of Plasmodium falciparum sexual stage antigens: Implications for the design of a transmission blocking vaccine. Immunol. Lett. 25, 83-86 (1990).

19. Barr, P. J. Recombinant Pfs25 protein of Plasmodium falciparum elicits malaria transmission-blocking immunity in experimental animals. J. Exp. Med. 174, 1203-1208 (2004).

20. Huang, W.-C. et al. A malaria vaccine adjuvant based on recombinant antigen binding to liposomes. Nat. Nanotechnol. 13, 1174-1181 (2018).

21. Li, Y. et al. Enhancing immunogenicity and transmission-blocking activity of malaria vaccines by fusing Pfs25 to IMX313 multimerization technology. Sci. Rep. 6, 18848 (2016).

22. McGuire, K. A., Miura, K., Wiethoff, C. M. \& Williamson, K. C. New adenovirus-based vaccine vectors targeting Pfs 25 elicit antibodies that inhibit Plasmodium falciparum transmission. Malar. J. 16, 254 (2017).

23. Qian, F. et al. Conjugating recombinant proteins to Pseudomonas aeruginosa ExoProtein A: a strategy for enhancing immunogenicity of malaria vaccine candidates. Vaccine 25, 3923-3933 (2007).

24. Chatterjee, S., Perignon, J. L., Marck, E. V. \& Druilhe, P. How reliable are models for malaria vaccine development? Lessons from irradiated sporozoite immunizations. J. Postgrad. Med. 52, 321-324 (2006).

25. Davis, H. L. Novel vaccines and adjuvant systems: the utility of animal models for predicting immunogenicity in humans. Hum. Vaccin. 4, 246-250 (2008).

26. Gerdts, V., Littel-van den Hurk, SvanD., Griebel, P. J. \& Babiuk, L. A. Use of animal models in the development of human vaccines. Future Microbiol. 2, 667-675 (2007).

27. Scally, S. W. et al. Molecular definition of multiple sites of antibody inhibition of malaria transmission-blocking vaccine antigen Pfs25. Nat. Commun. 8, 1568 (2017).

28. Lee, E. C. et al. Complete humanization of the mouse immunoglobulin loci enables efficient therapeutic antibody discovery. Nat. Biotechnol. 32, 356-363 (2014).

29. Miura, K. et al. Transmission-blocking activity induced by malaria vaccine candidates Pfs25/Pvs25 is a direct and predictable function of antibody titer. Malar. J. 6, 107 (2007).

30. Chichester, J. A. et al. Safety and immunogenicity of a plant-produced Pfs25 virus-like particle as a transmission blocking vaccine against malaria: a phase 1 dose-escalation study in healthy adults. Vaccine 36, 5865-5871 (2018).

31. Jones, R. M. et al. A novel plant-produced Pfs 25 fusion subunit vaccine induces long-lasting transmission blocking antibody responses. Hum. Vaccin. Immunother. 11, 124-132 (2015).

32. Tan, Y. C. et al. High-throughput sequencing of natively paired antibody chains provides evidence for original antigenic sin shaping the antibody response to influenza vaccination. Clin. Immunol. 151, 55-65 (2014).

33. Tan, Y. C. et al. Barcode-enabled sequencing of plasmablast antibody repertoires in rheumatoid arthritis. Arthritis Rheumatol. 66, 2706-2715 (2014).

34. Molecular Operating Environment (MOE). Chemical Computing Group Inc., 1010 Sherbrooke St. West, Suite \#910, Montreal, QC, Canada, H3A 2R7, 2016.

35. Saxena, A. K. Structure of Fab fragment of malaria transmission blocking antibody $2 \mathrm{~A} 8$ against $P$. vivax $\mathrm{P} 25$ protein. Int. J. Biol. Macromol. 50, 153-156 (2012).

36. Duan, H. et al. Glycan masking focuses immune responses to the HIV-1 CD4binding site and enhances elicitation of VRC01-class precursor antibodies. Immunity 49, 301-311 (2018). 
37. Zhou, T. et al. A neutralizing antibody recognizing primarily $\mathrm{N}$-linked glycan targets the silent face of the HIV envelope. Immunity 48, 500-513 (2018).

38. McLellan, J. S. et al. Structure-based design of a fusion glycoprotein vaccine for respiratory syncytial virus. Science 342, 592-598 (2013).

39. Yassine, H. M. et al. Hemagglutinin-stem nanoparticles generate heterosubtypic influenza protection. Nat. Med. 21, 1065-1070 (2015)

40. Impagliazzo, A. et al. A stable trimeric influenza hemagglutinin stem as a broadly protective immunogen. Science 349, 1301-1306 (2015).

41. Abbott, R. K. et al. Precursor frequency and affinity determine B cell competitive fitness in germinal centers, tested with germline-targeting HIV vaccine immunogens. Immunity 48, 133-146 (2018).

42. Jardine, J. G. et al. HIV-1 broadly neutralizing antibody precursor B cells revealed by germline-targeting immunogen. Science 351, 1458-1463 (2016)

43. Steichen, J. M. et al. HIV vaccine design to target germline precursors of glycan-dependent broadly neutralizing antibodies. Immunity 45, 483-496 (2016).

44. Havenar-Daughton, C. et al. The human naive B cell repertoire contains distinct subclasses for a germline-targeting HIV-1 vaccine immunogen. Sci. Transl. Med. 10, eaat0381 (2018).

45. Patra, K. P. et al. Alga-produced malaria transmission-blocking vaccine candidate Pfs 25 formulated with a human use-compatible potent adjuvant induces high-affinity antibodies that block Plasmodium falciparum infection of mosquitoes. Infect. Immun. 83, 1799-1808 (2015).

46. Radtke, A. J. et al. Adjuvant and carrier protein-dependent T-cell priming promotes a robust antibody response against the Plasmodium falciparum Pfs 25 vaccine candidate. Sci. Rep. 7, 40312 (2017).

47. Thompson, E. A. et al. TLR-adjuvanted nanoparticle vaccines differentially influence the quality and longevity of responses to malaria antigen Pfs25. JCI Insight 3, 120692 (2018).

48. Kundu, P. et al. Structural delineation of potent transmission-blocking epitope I on malaria antigen Pfs48/45. Nat. Commun. 9, 4458 (2018).

49. Kabsch, W. et al. XDS. Acta Crystallogr. D Biol. Crystallogr 66, 125-132 (2010).

50. McCoy, A. J. et al. Phaser crystallographic software. J. Appl. Crystallogr. 40 658-674 (2007).

51. Adams, P. D. et al. PHENIX: a comprehensive Python-based system for macromolecular structure solution. Acta Crystallogr. D Biol. Crystallogr. 66 213-221 (2010).

52. Emsley, P., Lohkamp, B., Scott, W. G. \& Cowtan, K. Features and development of Coot. Acta Crystallogr. D Biol. Crystallogr. 66, 486-501 (2010).

53. Morin, A. et al. Collaboration gets the most out of software. Elife 2, e01456 (2013).

54. Krissinel, E. \& Henrick, K. Inference of macromolecular assemblies from crystalline state. J. Mol. Biol. 372, 774-797 (2007).

55. Miura, K. et al. Qualification of standard membrane-feeding assay with Plasmodium falciparum malaria and potential improvements for future assays. PLoS ONE 8, e57909 (2013).

56. Miura, K. et al. Transmission-blocking activity is determined by transmissionreducing activity and number of control oocysts in Plasmodium falciparum standard membrane-feeding assay. Vaccine 34, 4145-4151 (2016).

\section{Acknowledgements}

This work was funded by PATH's Malaria Vaccine Initiative under Grant OPP1108403 from the Bill \& Melinda Gates Foundation. This research was undertaken, in part, thanks to funding from the Canada Research Chairs program (to J.-P.J.). S.W.S. was supported by a Hospital for Sick Children Lap-Chee Tsui postdoctoral fellowship and a Canadian Institutes of Health Research (CIHR) fellowship (FRN-396691). X-ray diffraction experiments were performed using beamline 08ID-1 at the Canadian Light Source, which is supported by the Canada Foundation for Innovation, Natural Sciences and Engineering Research Council of Canada, the University of Saskatchewan, the Government of Saskatchewan, Western Economic Diversification Canada, the National Research Council Canada, and the Canadian Institutes of Health Research. SMFA experiments were supported in part by the intramural program of the NIAID, NIH.

\section{Author contributions}

B.M., S.W.S., D.E., C.R.K. and J.-P.J. designed experiments; J.A.C. and S.S. provided clinical samples; B.M., K.M., S.W.S. and A.B. performed experiments; B.M., K.M., S.W.S., A.B., N.N., H.S., D.K., W.V., S.R., C.W., W.R.S., D.E., C.R.K. and J.-P.J. analyzed the data B.M., C.R.K. and J.-P.J. wrote the manuscript; and C.R.K. and J.-P.J. conceived the study.

\section{Additional information}

Supplementary Information accompanies this paper at https://doi.org/10.1038/s41467 019-11980-6.

Competing interests: The authors declare no competing interests.

Reprints and permission information is available online at http://npg.nature.com/ reprintsandpermissions/

Peer review information Nature Communications thanks the anonymous reviewers for their contribution to the peer review of this work. Peer reviewer reports are available.

Publisher's note Springer Nature remains neutral with regard to jurisdictional claims in published maps and institutional affiliations.

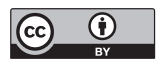

Open Access This article is licensed under a Creative Commons Attribution 4.0 International License, which permits use, sharing, adaptation, distribution and reproduction in any medium or format, as long as you give appropriate credit to the original author(s) and the source, provide a link to the Creative Commons license, and indicate if changes were made. The images or other third party material in this article are included in the article's Creative Commons license, unless indicated otherwise in a credit line to the material. If material is not included in the article's Creative Commons license and your intended use is not permitted by statutory regulation or exceeds the permitted use, you will need to obtain permission directly from the copyright holder. To view a copy of this license, visit http://creativecommons.org/ licenses/by/4.0/.

(C) The Author(s) 2019 